\title{
Network Pharmacology Analysis of the Identification of Phytochemicals and Therapeutic Mechanisms of Paeoniae Radix Alba for the Treatment of Asthma
}

\author{
Jingwei Wang, ${ }^{1,2,3}$ Ling Peng $\mathbb{D}^{4}{ }^{4}$ Lu Jin, ${ }^{1}$ Huiying Fu $\mathbb{D}^{1,2,3}$ and Qiyang Shou $\mathbb{D}^{1,2,3}$ \\ ${ }^{1}$ Second Clinical Medical College, Zhejiang Chinese Medical University, China \\ ${ }^{2}$ Academy of Chinese Medical Science, Zhejiang Chinese Medical University, China \\ ${ }^{3}$ Zhejiang Provincial Key Laboratory of Sexual Function of Integrated Traditional Chinese and Western Medicine, \\ Hangzhou, China \\ ${ }^{4}$ Department of Respiratory Disease, Zhejiang Provincial People's Hospital, Hangzhou, Zhejiang Province, China
}

Correspondence should be addressed to Huiying Fu; fhy131@126.com and Qiyang Shou; sqy133@126.com

Jingwei Wang and Ling Peng contributed equally to this work.

Received 29 June 2021; Accepted 17 August 2021; Published 14 September 2021

Academic Editor: Kai Wang

Copyright (C) 2021 Jingwei Wang et al. This is an open access article distributed under the Creative Commons Attribution License, which permits unrestricted use, distribution, and reproduction in any medium, provided the original work is properly cited.

Background. Paeoniae Radix Alba (PRA), the root of the plant Paeonia lactiflora Pall., has been suggested to play an important role for the treatment of asthma. A biochemical understanding of the clinical effects of Paeoniae Radix Alba is needed. Here, we explore the phytochemicals and therapeutic mechanisms via a systematic and comprehensive network pharmacology analysis. Methods. Through TCMSP, PubChem, GeneCards database, and SwissTargetPrediction online tools, potential targets of active ingredients from PRA for the treatment of asthma were obtained. Cytoscape 3.7.2 was used to determine the target of active ingredients of PRA. Target protein interaction (PPI) network was constructed through the STRING database. The Gene Ontology (GO) biological process and Kyoto Encyclopedia of Genes and Genes (KEGG) pathway enrichment analysis were analyzed through the biological information annotation database (DAVID). Results. Our results indicate that PRA contains 21 candidate active ingredients with the potential to treat asthma. The enrichment analysis of GO and KEGG pathways found that the treatment of asthma by PRA may be related to the process of TNF (tumor necrosis factor) release, which can regulate and inhibit multiple signaling pathways such as ceramide signaling. Conclusions. Our work provides a phytochemical basis and therapeutic mechanisms of PRA for the treatment of asthma, which provides new insights on further research on PRA.

\section{Introduction}

Asthma is a common condition due to chronic inflammation of the lower respiratory tract [1]. Asthma exhibits recurrent episodes of wheeze, cough, chest tightness, and shortness of breath. It arises from heterogenic gene-environment interactions which are not fully understood. Airway hyperresponsiveness, reversible airflow obstruction, and bronchial hyperresponsiveness are characteristic features of asthma [2]. Most asthma is associated with sensitization of the airways to common allergens. Upon exposure to allergen, early response was the result of activation of airway mast cells in an
IgE-dependent way with release of the rapidly acting granuleassociated preformed mediators, as histamine and tryptase, to contract airway smooth muscle, promote vascular leakage, and stimulate mucus secretion. Allergic response such as interleukins- (IL-) 4, IL-5, IL-6, and TNF- $\alpha$, in concert with the inflammatory mediators, stimulates the recruitment and activation of secondary effector cells, starting with neutrophils, followed by eosinophils and then T lymphocytes to cause late-phase airway narrowing and airway hyperresponsiveness [3]. This in turn produces long-term changes in the structure of the affected organs and substantial abnormalities in their function, which impact the quality of life. 
TABLE 1: Candidate active ingredients of PRA.

\begin{tabular}{|c|c|c|c|}
\hline Mol ID & Molecule name & $\mathrm{OB}(\%)$ & DL \\
\hline MOL001902 & $3 \beta, 23$-Dihydroxy-oleana-11,13(18)-dien-28-oic acid & 21.53 & 0.75 \\
\hline MOL001910 & 11Alpha,12alpha-epoxy-3beta-23-dihydroxy-30-norolean-20-en-28,12beta-olide & 64.77 & 0.38 \\
\hline MOL001911 & Albiflorin R1 & 21.29 & 0.82 \\
\hline MOL001912 & Albiflorin R1_qt & 26.18 & 0.34 \\
\hline MOL001918 & Paeoniflorgenone & 87.59 & 0.37 \\
\hline MOL001919 & $\begin{array}{l}\text { (3S,5R,8R,9R,10S,14S)-3,17-Dihydroxy-4,4,8,10,14-pentamethyl-2,3,5,6,7,9-hexahydro-1H- } \\
\text { cyclopenta[a]phenanthrene-15,16-dione }\end{array}$ & 43.56 & 0.53 \\
\hline MOL001921 & Lactiflorin & 49.12 & 0.8 \\
\hline MOL001924 & Paeoniflorin & 53.87 & 0.79 \\
\hline MOL001925 & Paeoniflorin_qt & 68.18 & 0.4 \\
\hline MOL001928 & Albiflorin_qt & 66.64 & 0.33 \\
\hline MOL001929 & Alexandrin & 20.63 & 0.62 \\
\hline MOL001930 & Benzoyl paeoniflorin & 31.27 & 0.75 \\
\hline MOL001933 & Oxypaeoniflorin & 21.88 & 0.78 \\
\hline MOL000211 & Mairin & 55.38 & 0.78 \\
\hline MOL000263 & Oleanolic acid & 29.02 & 0.76 \\
\hline MOL000358 & Beta-sitosterol & 36.91 & 0.75 \\
\hline MOL000359 & Sitosterol & 36.91 & 0.75 \\
\hline MOL000422 & Kaempferol & 41.88 & 0.24 \\
\hline MOL000492 & (+)-Catechin & 54.83 & 0.24 \\
\hline MOL000551 & Hederagenol & 22.42 & 0.74 \\
\hline MOL000357 & Sitogluside & 20.63 & 0.62 \\
\hline
\end{tabular}

Traditional Chinese medicine (TCM) has a long-lasting history of using herbal medicine in the treatment of various respiratory diseases [4]. There is increasing scientific evidence supporting the use of TCM for the treatment of asthma. Possible mechanisms include anti-inflammation, inhibition of airway smooth muscle contraction, and immunomodulation [5]. Paeoniae Radix Alba (Bai Shao, also known as Chinese peony), the root of the perennial herbaceous plant Paeonia lactiflora Pall. of the buttercup family, has the effects of nourishing blood and regulating menstruation, restraining yin and antiperspirant, softening liver and relieving pain, and suppressing liver-yang [6]. Our previous studies investigated the antiallergic role of total glucosides of peony, indicating that total glucosides of peony have an effect on the allergic reaction of mice [7]. Thus, we speculate that the total glucosides of peony should have a therapeutic effect on asthma. In this study, network pharmacology was utilized to analyse the active ingredients, drug targets, and key pathways of PRA for the treatment of asthma.

\section{Methods}

2.1. Composition of Chinese Medicine White Peony. TCMSP database was used to obtain the pharmaceutical ingredients with "Paeoniae Radix Alba" as the key word. The ingredients that meet the criteria with oral biological degree (OB) $>20 \%$ and drug - like properties (DL) $>0.18$ at the same time were selected as the active ingredients of PRA. Effective active ingredients of PRA contained in the database were queried and screened.

2.2. Prediction of Small Molecule Target Protein. Based on the TCMSP and DrugBank databases, target proteins corresponding to small molecules were sorted out, while components without target proteins and duplicate targets were deleted. Using the UniProt (http://www.uniprot.org/) database, screened targets are converted into gene names, full protein names are corresponded to the gene abbreviations, and the component target-gene data table is made.

2.3. Chemical-Gene Network Analysis. According to the chemical molecule-gene relationship obtained by the analysis, component target-gene data were imported into the Cytoscape 3.7.2 software. The data were analyzed and displayed graphically in the form of a network diagram. Different nodes are used to represent data types, and connections are used to represent interaction relationships. Topological analysis is performed, and the core target of PRA is selected according to the node degree. The node degree is one of the main data methods to determine the key nodes. The higher the node degree, the more important the role of the node in the network.

2.4. Asthma Gene Acquisition and Analysis. The UniProt database (https://www.uniprot.org/) and TTD data (https:// db.idrblab.org/ttd/) database were searched for asthmarelated targets. Duplicate targets were deleted, and information acquired were collected. 
TABLE 2: Target-gene pairs of active ingredients of PRA.

\begin{tabular}{|c|c|}
\hline Target gene & Gene name \\
\hline $26 \mathrm{~S}$ proteasome non-ATPase regulatory subunit 3 & PSMD3 \\
\hline 5-Hydroxytryptamine $2 \mathrm{~A}$ receptor & HTR2A HTR2 \\
\hline 5-Hydroxytryptamine receptor $3 \mathrm{~A}$ & HTR3A \\
\hline Acetylcholinesterase & ACHE \\
\hline Activator of $90 \mathrm{kDa}$ heat shock protein ATPase homolog 1 & AHSA1 \\
\hline Aldo-keto reductase family 1 member $\mathrm{C} 3$ & AKR1C3 \\
\hline Alpha-1D adrenergic receptor & ADRA1B \\
\hline Androgen receptor & $\mathrm{AR}$ \\
\hline Antileukoproteinase & SLPI \\
\hline Apoptosis regulator BAX & BAX \\
\hline Apoptosis regulator Bcl-2 & BCL2 \\
\hline Arachidonate 5-lipoxygenase & ALOX5 \\
\hline Aryl hydrocarbon receptor & AHR \\
\hline Beta-2 adrenergic receptor & ADRB2 ADRB2R, B2AR \\
\hline Beta-lactamase & blaC \\
\hline Calmodulin & CAMKK2 \\
\hline Caspase-3 & CASP3 \\
\hline Caspase- 8 & CASP8 \\
\hline Caspase- 9 & CASP9 MCH6 \\
\hline Catalase & CAT \\
\hline Cell division control protein 2 homolog & $\mathrm{CDC} 42$ \\
\hline CGMP-inhibited $3^{\prime}, 5^{\prime}$-cyclic phosphodiesterase A & PDE3A \\
\hline Coagulation factor VII & F7 \\
\hline Coagulation factor $\mathrm{Xa}$ & F10 \\
\hline Cytochrome P450 1A1 & CYP1A1 \\
\hline Cytochrome P450 1A2 & CYP1A2 \\
\hline Cytochrome P450 1B1 & CYP1B1 \\
\hline Cytochrome P450 3A4 & CYP3A4 \\
\hline Cytochrome P450-cam & camC \\
\hline Dipeptidyl peptidase IV & DPP4 \\
\hline DNA topoisomerase II & TOP2 \\
\hline Dopamine D1 receptor & DRD5 DRD1B, DRD1L2 \\
\hline E-Selectin & SELE \\
\hline Estrogen receptor & ESR1 \\
\hline Gamma-aminobutyric acid receptor subunit alpha-1 & GABRA1 \\
\hline Gamma-aminobutyric-acid receptor alpha-2 subunit & GABRA2 \\
\hline Gamma-aminobutyric-acid receptor alpha-3 subunit & GABRA3 \\
\hline Gamma-aminobutyric-acid receptor alpha-5 subunit & GABRA5 \\
\hline Glutathione S-transferase Mu 1 & GSTM1 \\
\hline Glutathione S-transferase $\mathrm{Mu} 2$ & GSTM2 \\
\hline Glutathione S-transferase P & GSTP1 \\
\hline Heat shock protein HSP 90 & HSP90AA1 HSP90A, HSPC1, HSPCA \\
\hline Heme oxygenase 1 & HMOX1 HO, HO1 \\
\hline Hyaluronan synthase 2 & HAS2 \\
\hline Inhibitor of nuclear factor kappa-B kinase subunit beta & IKBKB \\
\hline Insulin receptor & INSR \\
\hline Intercellular adhesion molecule 1 & ICAM1 \\
\hline Interleukin-6 & IL6 \\
\hline
\end{tabular}


TABLE 2: Continued.

\begin{tabular}{|c|c|}
\hline Target gene & Gene name \\
\hline Interstitial collagenase & MMP1 \\
\hline Lipopolysaccharide-binding protein & LBP \\
\hline Microtubule-associated protein 2 & Map2 \\
\hline Mineralocorticoid receptor & $\mathrm{NR} 3 \mathrm{C} 2$ \\
\hline Mitogen-activated protein kinase 8 & MAPK8 \\
\hline Monocyte differentiation antigen CD14 & CD14 \\
\hline mRNA of PKA catalytic subunit C-alpha & PRKACA \\
\hline Muscarinic acetylcholine receptor M1 & CHRM1 \\
\hline Muscarinic acetylcholine receptor M2 & CHRM2 \\
\hline Muscarinic acetylcholine receptor M3 & CHRM3 \\
\hline Muscarinic acetylcholine receptor M4 & CHRM4 \\
\hline Mu-type opioid receptor & OPRM1 MOR1 \\
\hline NAD(P)H dehydrogenase [quinone] 1 & NQO1 DIA4, NMOR1 \\
\hline Neuronal acetylcholine receptor protein, alpha-7 chain & CHRNA7 NACHRA7 \\
\hline Neuronal acetylcholine receptor subunit alpha-2 & CHRNA2 \\
\hline Nitric-oxide synthase, inducible & NOS2 \\
\hline Nitric-oxide synthase, endothelial & NOS3 \\
\hline Nuclear receptor coactivator 2 & NCOA2 \\
\hline Nuclear receptor subfamily 1 group I member 2 & NR1I2 \\
\hline Pancreatic alpha-amylase & $\mathrm{AMY} 2 \mathrm{~A}$ \\
\hline Peroxidase C1A & PRXC1A \\
\hline Peroxisome proliferator activated receptor gamma & PPARG \\
\hline Phosphatidylinositol-4,5-bisphosphate 3-kinase catalytic subunit, gamma isoform & PIK3CG \\
\hline Potassium voltage-gated channel subfamily $\mathrm{H}$ member 2 & KCNH2 ERG, ERG1, HERG \\
\hline Progesterone receptor & PGR \\
\hline Prostaglandin $\mathrm{G} / \mathrm{H}$ synthase 1 & PTGS1 \\
\hline Prostaglandin $\mathrm{G} / \mathrm{H}$ synthase 2 & PTGS2 \\
\hline Protein kinase C-alpha type & PRKCA \\
\hline RAC-alpha serine/threonine-protein kinase & AKT1 \\
\hline Retinoic acid receptor RXR-alpha & RXRA \\
\hline Serine/threonine-protein phosphatase $2 \mathrm{~B}$ catalytic subunit alpha isoform & PPP3CA \\
\hline Serum paraoxonase/arylesterase 1 & PON1 \\
\hline Signal transducer and activator of transcription 1-alpha/beta & STAT1 \\
\hline Sodium channel protein type 5 subunit alpha & SCN5A \\
\hline Sodium-dependent noradrenaline transporter & SLC6A2 \\
\hline Solute carrier family 2 , facilitated glucose transporter member 4 & SLC2A4 \\
\hline Thrombin & F2 \\
\hline Transcription factor AP-1 & JUN \\
\hline Transcription factor p65 & RELA \\
\hline Transforming growth factor beta-1 & TGFB1 \\
\hline Trypsin-1 & PRSS1 \\
\hline Tumor necrosis factor & TNF \\
\hline
\end{tabular}

2.5. Gene Pathway and Function Analysis. The DAVID database (https://david.ncifcrf.gov/) and Metascape database (https://metascape.org/) were used to analyze the GO function and KEGG pathway enrichment analysis of the potential targets of PRA for the treatment of asthma. GOTERM_BP_DIRECT (biological process), GOTERM_CC_DIRECT (cell composition), and GOTERM_MF_DIRECT (molecular function) are saved in Gene_Ontology; KEGG_PATH-WAY was saved in Pathways.

\section{Results}

3.1. Screening of Active Ingredients of Paeonia. Oral administration is the most common route of traditional Chinese 


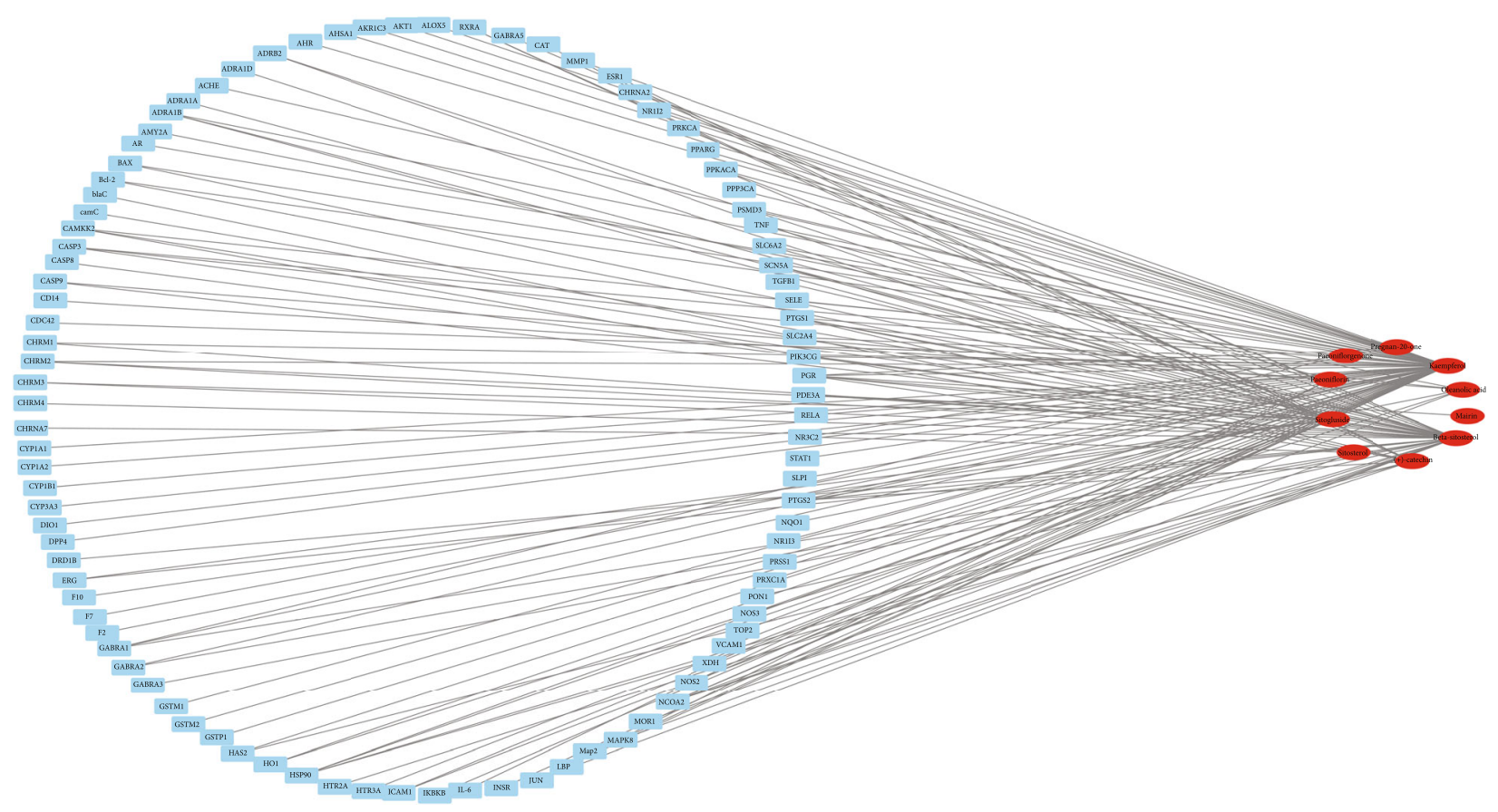

FIgUre 1: Part of main active ingredients of PRA.

TABLE 3: Degree of top 5 chemicals and target proteins of PRA.

\begin{tabular}{lccc}
\hline Gene & Degree & Molecule & Degree \\
\hline PGR & 6 & Kaempferol & 63 \\
NCOA2 & 5 & Beta-sitosterol & 39 \\
HSP90 & 4 & Sitogluside & 17 \\
PTGS1 & 4 & $(+)$-Catechin & 11 \\
PTGS2 & 4 & Oleanolic acid & 6 \\
\hline
\end{tabular}

Medicine preparations. OB is an effective index to evaluate the clinical efficacy of traditional Chinese medicine. Through the TCMSP database, combined with "OB $>20 \%$ " and "DL $>0.18$ ", 21 active ingredients in PRA that can be orally absorbed were screened out, as shown in Table 1.

3.2. Target Prediction Results of Active Ingredients in White Peony. Reverse docking through the SwissTargetPrediction website was used using "Homo sapiens." Target proteins of the active ingredients were screened, and gene target corresponding to each ingredient was acquired. A total of 147 targets after removing 90 targets were sort out, and the target-gene pairs were obtained, which are shown in Table 2.

3.3. Small Molecule Target Protein. Based on the TCMSP and DrugBank databases, the target protein relationship of each chemical small molecule after integration was obtained. Figure 1 is a network diagram of the effective small chemical molecules and target proteins contained in PRA. The network diagram contains 42 nodes ( 10 small chemical molecules and 96 target proteins) and 145 edges. Table 3 shows the degree table of the small chemical molecules and target proteins of PRA. In this network, kaempferol with the highest degree of connectivity regulates 63 target proteins, followed by beta-sitosterol, sitogluside, $(+)$-catechin, etc. The target protein with the highest degree of connectivity is PGR, which is regulated by 6 small chemical molecules, followed by NCOA2, HSP90, PTGS1, PTGS2, and so on.

3.4. Functional Analysis. Based on the DAVID tool, the GO function and KEGG pathway enrichment analysis of the target genes of PRA were performed, and the significance threshold was selected as the correction (Benjamini $P$ value $<0.01)$. Figure 2 is the GO analysis diagram. The most significant biological processes are cellular response to organic cyclic compound, response to lipopolysaccharide, and response to toxic substance. Figure 3 shows the enrichment of the first 30 pathways. The most significant pathways are toxoplasmosis, Toll-like receptor signaling, osteoclast differentiation, apoptosis, and the Chagas disease. The interaction between related proteins can be classified into positive regulation of vasoconstriction, toxoplasmosis, and estrogen metabolic process, as shown in Figure 4, among which the most widely involved is the vasoconstriction.

3.5. Key Gene Screening of Asthma. We searched for disease targets in databases as Delegated Database Tree (DDT), DrugBank, and DisGeNET, and a total of 153 asthma-related targets were obtained. The intersection of these targeted genes with the previous targets of the active substance of PRA was analyzed. Finally, 13 genes are both target genes (Table 4). KEGG enrichment analysis was performed on these 13 key genes, and $P<0.05$ was chosen as the threshold, which is shown in Table 5. Through the enrichment analysis of the coacting targets, the red node in Figure 5 indicates the key upregulated gene of asthma, which only contains TNF. We believe that only TNF is the key gene in the treatment of asthma with white peony drugs, and some downregulated 


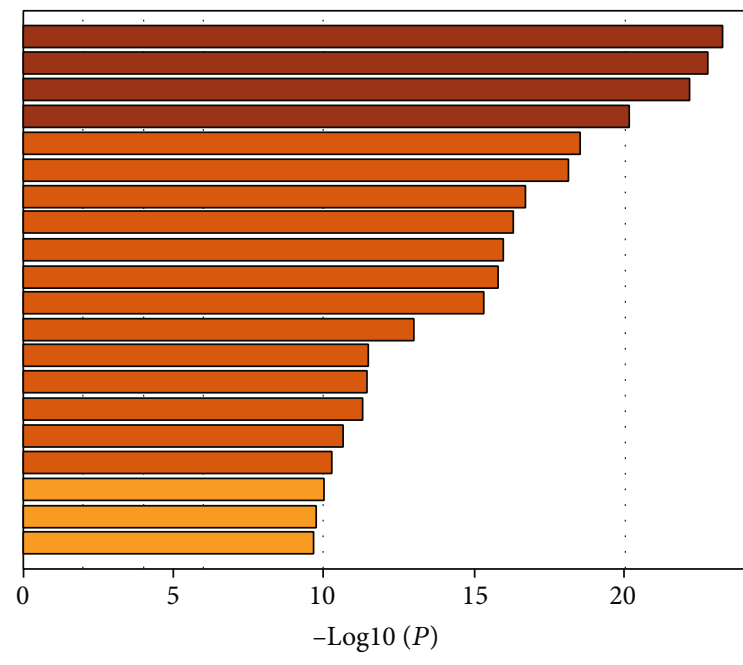

GO:0071407: cellular response to organic cyclic compound GO:0032496: response to lipopolysacchride GO:0009636: response to toxic substance

hsa 04933: AGE-RAGE signaling pathway in diabetic complications GO:0009410: response to xenobiotic stimulus

GO:0008015: blood circulation

GO:0032870: cellular response to hormone stimulus

GO:1901699: cellular response to nitrogen compound M115: PID REG GR pathway

GO:0072593: reactive oxygen species metabolic process

GO:0099536: synaptic signaling

GO:0000302: response to reactive oxygen species

GO:0019932: second-messenger-mediated signaling

GO:0035690: cellular response to drug

GO:0001676: long-chain fatty acid metabolic process

M99: PID TXAS pathway

GO:0050673: epithelial cell proliferation

GO:1901654: response to ketone

hsa01524: platinum drug resistance

hsa04725: cholinergic synapse

(a)

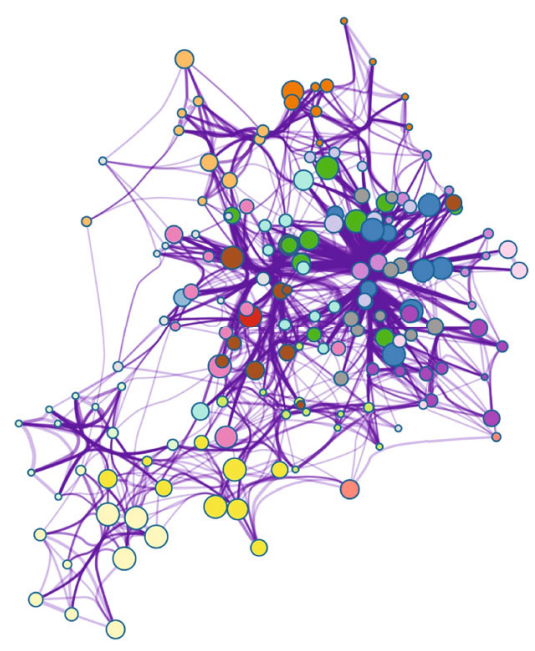

Cellular response to organic cycllic compound

Response to lipopolysaccharide

Response to toxic substance

AGE-RAGE signaling pathway in diabetic complicatic

Response to xenobiotic stimulus

Blood circulation

Cellular response to hormone stimulus

Cellular response to nitrogen compound

PID REG GR PATHWAY

Reaction oxygen species metabolic process

Synaptic signaling

Response to reactive oxygen species

Second-messenger-mediated signaling

Cellular response to drug

Long-chain fatty acid metabolic process

PID TXA2PATHWAY

Epithelial cell proliferation

Response to ketone

Platinum drug resistance

Cholinergic synapse

(b)

Figure 2: Graph of the GO function analysis bar and PPI. (a) GO analysis. (b) PPI network. 


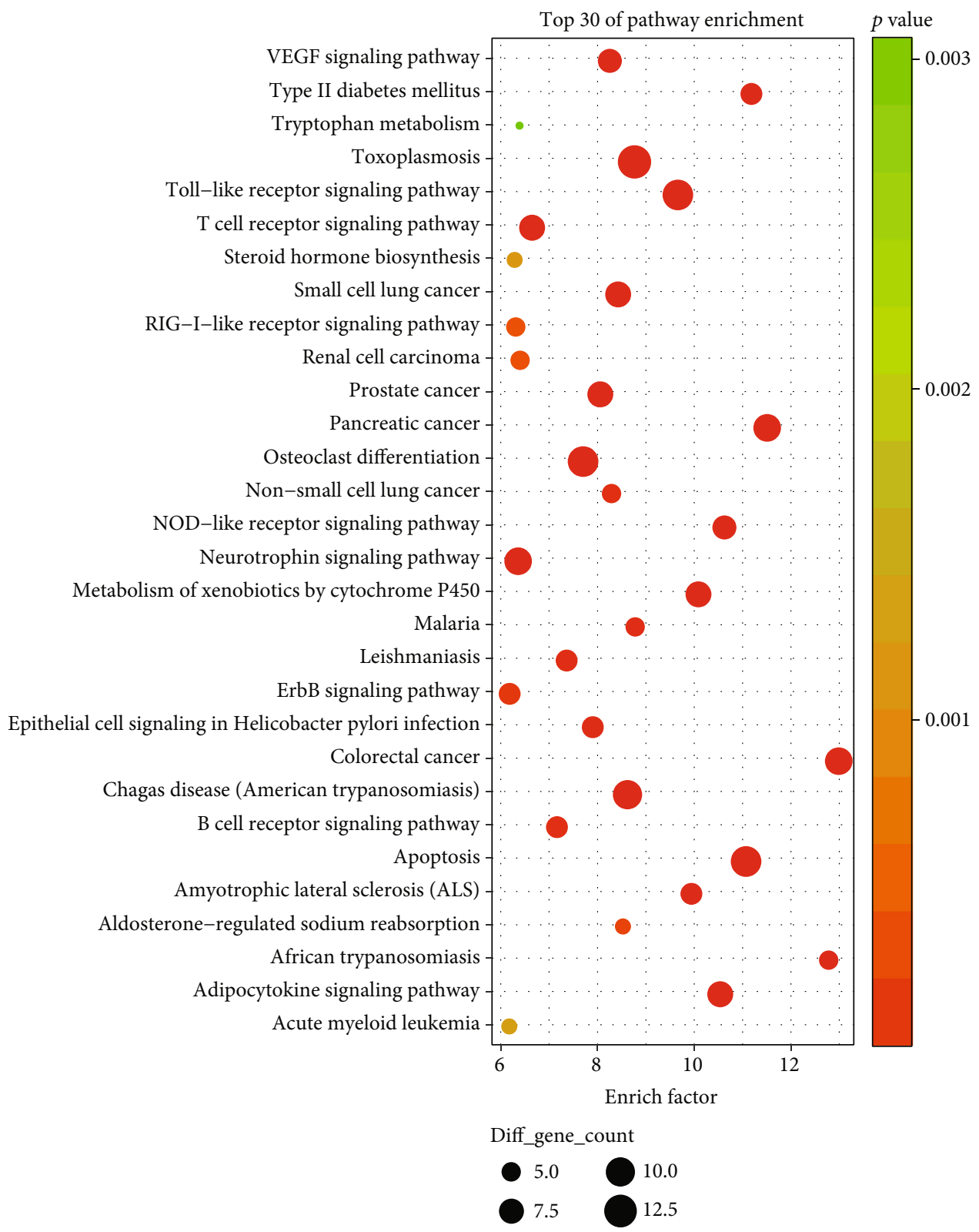

Figure 3: KEGG-enriched bubble chart.

genes such as IL-4 and IL-13 will affect the regulation of asthma.

\section{Discussion}

Asthma is a chronic disease characterized by inflammation hyperreactivity of the airways [8]. It is a complex syndrome with many clinical phenotypes in adults and children, which consists different phenotypes that share common features with distinct etiologies and pathophysiological pathways leading to disease [9]. The inhaled allergen encounters antigen presenting cells (APCs) in the airways. After recognizing the antigen and being activated by APCs, naïve T cells differentiate into Th2 cells, and the activated Th2 cells stimulate B cells to form IgE. IgE molecules then bind to IgE receptors on mast cells. The IgE crosslinking of allergens and mast cells will release biologically active mediators (histamine and leukotrienes) through degranulation, leading to direct symptoms of allergies [10]. Mast cells also release chemokines, which help the recruitment of inflammatory cells, especially eosinophils, which secret IL-5 to promote their proliferation and differentiation from bone marrow progenitor cells [11]. The activated eosinophils will release toxic particles and oxygen-free radicals, which leads to tissue damage and promotes the development of chronic inflammation.

The human TNF- $\alpha$ gene is about $2.76 \mathrm{~kb}$ in length and consists 4 exons and 3 introns. It is closely linked to the MHC gene group and is located on the $6^{\text {th }}$ and $17^{\text {th }}$ chromosomes, respectively. The natural form of TNF- $\alpha$ exerts its biological effects is a homotrimer. TNF- $\alpha$ is mainly produced and secreted by activated alveolar macrophages with a variety of biologically active cytokines, which can stimulate 

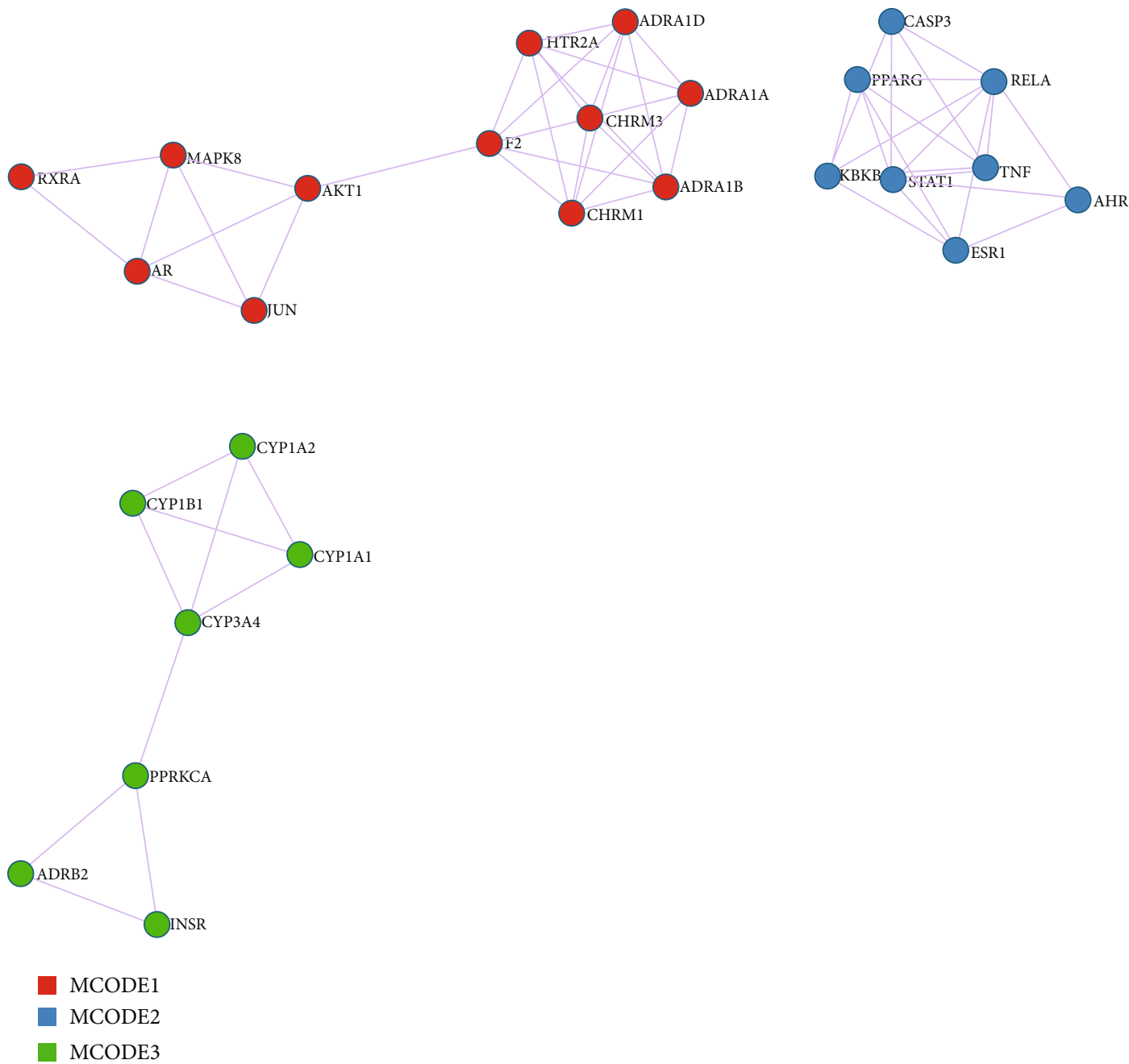

FIgURE 4: Protein interaction network of active ingredient in PRA.

TABle 4: Common target genes of PRA and asthma.

\section{Comment target genes}

Arachidonate 5-lipoxygenase (5-LOX)

DNA topoisomerase II (TOP2)

E-selectin (SELE)

Muscarinic acetylcholine receptor M2 (CHRM2)

Muscarinic acetylcholine receptor M3 (CHRM3)

Muscarinic acetylcholine receptor M4 (CHRM4)

Neuronal acetylcholine receptor alpha-7 (CHRNA7)

Nitric-oxide synthase inducible (NOS2)

Prostaglandin G/H synthase (COX/PTGS1)

Prostaglandin G/H synthase 2 (COX-2/PTGS2)

Tumor necrosis factor (TNF)

Heat shock protein 90 (HSP90)

neutrophils to cause degranulation and an increase in respiratory burst activity. TNF- $\alpha$ can promote the adhesion of neutrophils to endothelial cells [12], and it can also promote the activation of monocytes and accelerate the expression of
IL-2 receptors [13]. The phagocytic function of alveolar macrophages causes damage, which stimulates the activation of inflammatory mediators and induces the body to produce asthma symptoms. The asthma pathway map of our study indicates that TNF- $\alpha$ is the key target of PRA for the treatment of asthma. Among them, TNF-related active ingredients are kaempferol and paeoniflorin. Kaempferol suppresses eosinophil infiltration and airway inflammation in airway epithelial cells and in mice with allergic asthma through inhibition of TNF- $\alpha$ [14]. Kaempferol can inhibit ceramide signaling pathway, which reduce the release of TNF- $\alpha$, reduce the transcription of MMP genes, and reduce the expression of MMP. Due to the reduced expression of $\mathrm{MMP}$, it can effectively protect the extracellular membrane (ECM) and basement membrane in the lung from dissolution and avoid asthma symptoms [15]. Paeoniflorin attenuates adipocyte lipolysis and inhibits the phosphorylation of ERK, JNK, and IKK stimulated by TNF- $\alpha$ [16]. Thus, previous work on the compounds correlates with the results of our bioinformatic analysis of network pharmacology exploration of asthma and PRA.

Furthermore, there are many other genes which play roles in the coacting target genes, such as CHRM, COX, 
TABLE 5: KEGG pathway enrichment analysis of key 13 genes (part).

\begin{tabular}{lcr}
\hline Term & Input & $P$ value \\
\hline P00003:Alzheimer disease-amyloid secretase pathway & 4 & $5.76 E-09$ \\
hsa04725:cholinergic synapse & 4 & $5.33 E-08$ \\
hsa04080:neuroactive ligand-receptor interaction & 5 & $6.40 E-08$ \\
hsa00590:arachidonic acid metabolism & 3 & $1.33 E-06$ \\
hsa04668:TNF signaling pathway & 3 & $7.12 E-06$ \\
hsa04726:serotonergic synapse & 3 & $7.69 E-06$ \\
hsa05310:asthma & 1 & 0.0107 \\
H00079:asthma & 1 & 0.00368 \\
\hline
\end{tabular}

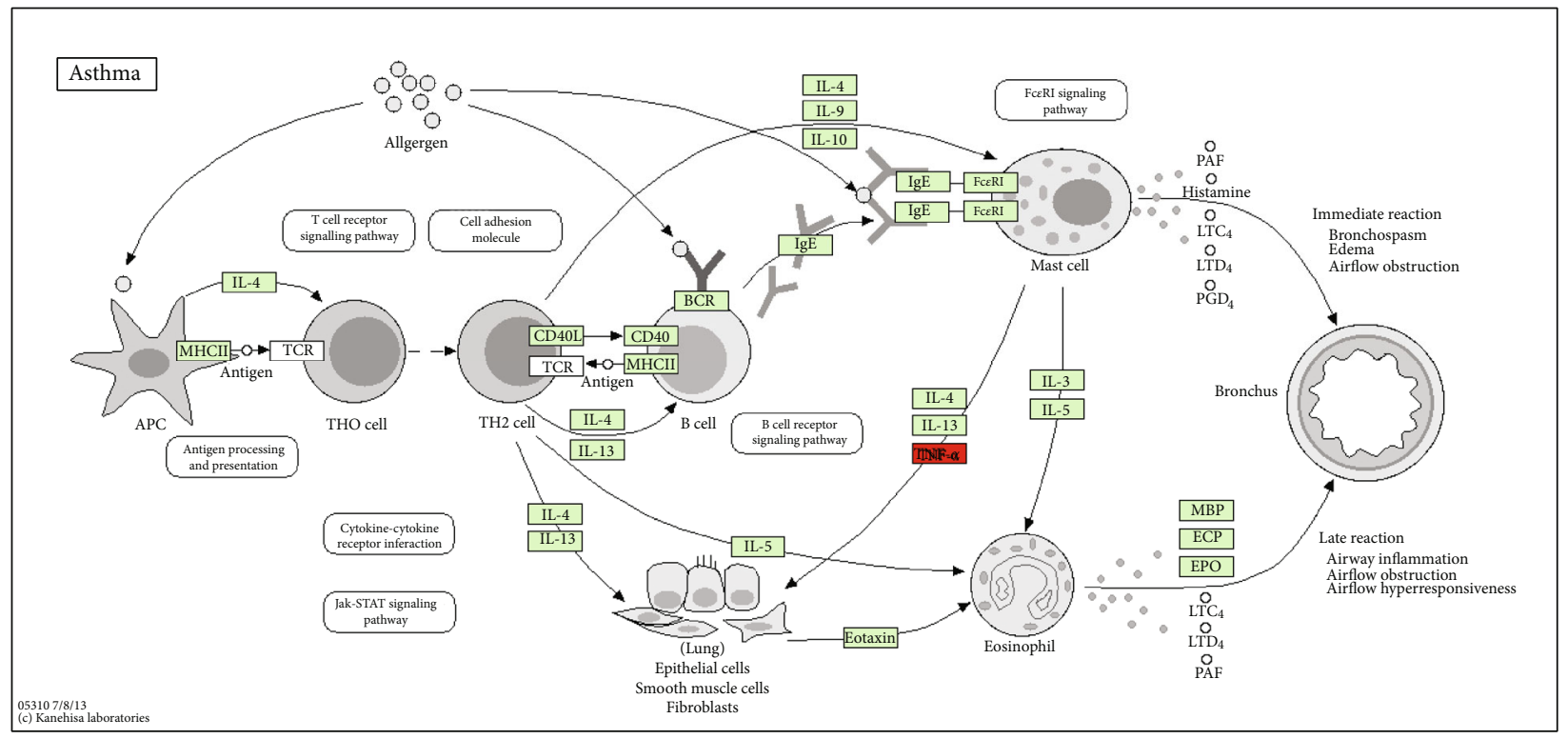

FIgURE 5: The pathway map of asthma.

and PTGS2. Kaempferol has anti-inflammatory effects by inhibiting interleukin-4 (IL-4) kinase and downregulating the NF- $\kappa$ B pathway [17]. By inhibiting IL-4 expression and cyclooxygenase 2 (COX2), kaempferol has a significant inhibitory effect on NADPH oxidase activity. When kaempferol reduces the reactive oxygen species (ROS) through the direct binding of NADPH oxidase, it can promote COX2 induction, thereby inhibiting epithelial thickening, indicating that this component can have a better adjuvant therapeutic effect on respiratory diseases through anti-inflammatory effects [18].

There are many highly sensitive cough receptors in the throat and trachea. When the mucosa of respiratory tract is stimulated by foreign bodies or secretions in the respiratory tract, it will produce a cough response through a series of neural reflexes, with the intention of expelling the secretions in the respiratory tract. A series of mechanical or chemical stimuli, such as the inhalation of harmful gases, the invasion of pathogenic microorganisms, the inflammatory factors produced by airway inflammation, and the contraction of airway smooth muscles, can all be used as inducements to cause the receptors to transmit signals to the cough center after the body is stimulated. Through network pharmacological analysis, it is found that PRA can act on key targets in cough response-related pathways. Beta-sitosterol and sitogluside, the active ingredients in PRA, can regulate the concentration of $\mathrm{Ca}^{2+}$ through the calcium ion pathway and inhibit the activation of CHRM2 and CHRM3 on the bronchial smooth muscle, thereby inhibiting the contraction of the bronchial smooth muscle and reducing cough [19]. The stimulus received by the receptors, in turn, plays a role in relieving cough. In addition, the $\mathrm{Ca}^{2+}$ produced by this reaction can directly bind to ion channels and can indirectly participate in the process of other signaling pathways related to the cough and asthma reaction [20].

Asthma is mainly related to allergic inflammation and neurological reactions. When the mucous membrane of the respiratory tract tissue is stimulated by allergens, it will promote the production of large amounts of IgE antibodies, triggering type I allergies. IgE high-affinity receptor "FceRI" is expressed on the surface of a variety of immune cells (mast 
cells, B cells, eosinophils, etc.). The massively secreted IgE can trigger allergic inflammation cascades by activating inflammatory cells, leading to airway smooth muscles contract, triggering asthma. Through network pharmacology analysis, it is found that $\beta$-sitosterol can bind to key targets on the pathways related to asthma response [21]. Chan et al. found through research that the PTGS2 gene is closely related to the inflammation of the asthmatic response, and the cyclooxygenase encoded by the PTGS2 gene has an airway remodeling effect [22]. Kaempferol can slow down the asthma response by breaking down fat or regulating fatty acid metabolism [23]. From the molecular mechanism of our result, it is found that PRA has a strong inhibitory effect on the inflammation of the respiratory system. Further studies are warranted to clarify the role of PRA in the treatment of asthma via the TNF- $\alpha$ signaling pathway.

In summary, this study used network pharmacology to analyze the main active ingredients of PRA. The main active ingredients and their possible molecular mechanisms for the treatment of asthma have been clarified. These results provide a theoretical basis of PRA for the treatment of asthma and provide a scientific basis for its better clinical application.

\section{Data Availability}

Source data of this study is derived from the public repositories, as indicated in "Methods" of the manuscript. All data that support the findings of this study is available from the corresponding authors upon reasonable request.

\section{Conflicts of Interest}

The authors declare that they have no conflicts of interest.

\section{Authors' Contributions}

All authors listed have made a substantial, direct, and intellectual contribution to the work and approved it for publication. Jingwei Wang and Ling Peng contributed equally to this work. Jingwei Wang and Ling Peng are co-first authors.

\section{Acknowledgments}

This work was supported by the National Natural Science Foundation of China (Nos. 81573677, 81673645, and 81873047), Opening Project of Zhejiang Provincial Firstrate Subject (Traditional Chinese Medicine) of Zhejiang Chinese Medical University (No. Ya2017012), and Scientific Funding of Zhejiang Chinese Medical University (No. 2020ZZ11).

\section{References}

[1] A. Papi, C. Brightling, S. Pedersen, and H. Reddel, "Asthma," The Lancet, vol. 391, 2018.

[2] T. Boonpiyathad, Z. Sözener, P. Satitsuksanoa, and C. Akdis, "Immunologic mechanisms in asthma," Seminars in Immunology, vol. 46, p. 101333, 2019.
[3] S. Kany, J. T. Vollrath, and B. Relja, "Cytokines in inflammatory disease," International Journal of Molecular Sciences, vol. 20, no. 23, p. 6008, 2019.

[4] H. Chan and T. Ng, "Traditional Chinese medicine (TCM) and allergic diseases," Current Allergy and Asthma Reports, vol. 20, no. 11, 2020.

[5] X.-M. Li and L. Brown, "Efficacy and mechanisms of action of traditional Chinese medicines for treating asthma and allergy," The Journal of allergy and clinical immunology, vol. 123, no. 2, pp. 297-306, 2009.

[6] Y. Q. Tan, H. W. Chen, J. Li, and Q. J. Wu, "Efficacy, chemical constituents, and pharmacological actions of Radix Paeoniae Rubra and Radix Paeoniae Alba," Frontiers in Pharmacology, vol. 11, p. 1054, 2020.

[7] Q. Shou, J. Lang, L. Jin et al., "Total glucosides of peony improve ovalbumin-induced allergic asthma by inhibiting mast cell degranulation," Journal of Ethnopharmacology, vol. 244, p. 112136, 2019.

[8] S. T. Holgate, S. Wenzel, D. S. Postma, S. T. Weiss, H. Renz, and P. D. Sly, "Asthma," Nature Reviews. Disease Primers, vol. 1, p. 15025, 2015.

[9] M. E. Kuruvilla, F. E. Lee, and G. B. Lee, "Understanding asthma phenotypes, endotypes, and mechanisms of disease," Clinical Reviews in Allergy and Immunology, vol. 56, pp. 219-233, 2019.

[10] S. J. Galli and M. Tsai, "IgE and mast cells in allergic disease," Nature Medicine, vol. 18, pp. 693-704, 2012.

[11] M. Krystel-Whittemore, K. N. Dileepan, and J. G. Wood, "Mast cell: a multi-functional master cell," Frontiers in Immunology, vol. 6, p. 620, 2015.

[12] W. Y. Sun, S. M. Pitson, and C. S. Bonder, "Tumor necrosis factor-induced neutrophil adhesion occurs via sphingosine kinase-1-dependent activation of endothelial \{alpha\}5 5 beta 1 integrin," The American Journal of Pathology, vol. 177, pp. 436-446, 2010.

[13] N. Parameswaran and S. Patial, "Tumor necrosis factor-alpha signaling in macrophages," Critical Reviews in Eukaryotic Gene Expression, vol. 20, pp. 87-103, 2010.

[14] J. H. Gong, D. Shin, S. Y. Han, J. L. Kim, and Y. H. Kang, "Kaempferol suppresses eosionphil infiltration and airway inflammation in airway epithelial cells and in mice with allergic asthma," The Journal of Nutrition, vol. 142, pp. 47-56, 2012.

[15] C. P. Vieira, L. P. de Oliveira, M. B. Da Silva et al., "Role of metalloproteinases and TNF-alpha in obesity-associated asthma in mice," Life Sciences, vol. 259, p. 118191, 2020.

[16] P. Kong, R. Chi, L. Zhang, N. Wang, and Y. Lu, "Effects of paeoniflorin on tumor necrosis factor-alpha-induced insulin resistance and changes of adipokines in 3T3-L1 adipocytes," Fitoterapia, vol. 91, pp. 44-50, 2013.

[17] B. Li, B. Luo, L. Jiang, and C. Jiang, "Kaempferol nanoparticles achieve strong and selective inhibition of ovarian cancer cell viability," International Journal of Nanomedicine, vol. 7, pp. 3951-3959, 2012.

[18] D. Shin, S.-H. Park, Y.-J. Choi et al., "Dietary compound kaempferol inhibits airway thickening induced by allergic reaction in a bovine serum albumin-induced model of asthma," International Journal of Molecular Sciences, vol. 16, pp. 29980-29995, 2015.

[19] E. A. Townsend, M. A. Thompson, C. M. Pabelick, and Y. S. Prakash, "Rapid effects of estrogen on intracellular $\mathrm{Ca} 2+$ 
regulation in human airway smooth muscle," American Journal of Physiology. Lung Cellular and Molecular Physiology, vol. 298, pp. L521-L530, 2010.

[20] M. A. Valverde, G. Cantero-Recasens, A. Garcia-Elias, C. Jung, A. Carreras-Sureda, and R. Vicente, "Ion channels in asthma," The Journal of Biological Chemistry, vol. 286, pp. 32877-32882, 2011.

[21] Y. Sun, L. Gao, W. Hou, and J. Wu, "Beta-sitosterol alleviates inflammatory response via inhibiting the activation of ERK/p38 and NF-kappaB pathways in LPS-exposed BV2 cells," BioMed Research International, vol. 2020, Article ID 7532306, 2020.

[22] I. H. S. Chan, N. L. S. Tang, T. F. Leung et al., "Association of prostaglandin-endoperoxide synthase 2 gene polymorphisms with asthma and atopy in Chinese children," Allergy, vol. 62, pp. 802-809, 2007.

[23] L. Thors, M. Belghiti, and C. J. Fowler, "Inhibition of fatty acid amide hydrolase by kaempferol and related naturally occurring flavonoids," British Journal of Pharmacology, vol. 155, pp. 244-252, 2008. 